\title{
Potassium Voltage-Gated Channel Subfamily KQT Member 1
}

National Cancer Institute

\section{Source}

National Cancer Institute. Potassium Voltage-Gated Channel Subfamily KQT Member 1. NCI Thesaurus. Code C75624.

Potassium voltage-gated channel subfamily KQT member 1 (676 aa, $75 \mathrm{kDa}$ ) is encoded by the human KCNQ1 gene. This protein is involved in cardiac repolarization through modulation of potassium ion transport. 